\title{
Refining the role of epicardial adipose tissue in non-alcoholic fatty liver disease
}

\author{
Mohammed Eslam $^{1} \cdot$ Jacob George ${ }^{1}$
}

Received: 12 August 2019 / Accepted: 18 September 2019 / Published online: 4 October 2019

(c) Asian Pacific Association for the Study of the Liver 2019

\section{Introduction}

Obesity is a rapidly growing public health challenge, with global prevalence having increased substantially over four decades, from less than $3 \%$ and $6 \%$ in 1975 , to $11 \%$ and $15 \%$ in 2016, among male and female, respectively [1]. This development, together with concomitant increases in diabetes, has fuelled a dramatic escalation in the prevalence of non-alcoholic fatty liver disease (NAFLD). NAFLD is now a leading cause of chronic liver disease, and afflicts about a quarter of the global population [2].

Although it is accepted that visceral adipose tissue (VAT) is an independent risk factor for NAFLD and NAFLD related fibrosis [3, 4], there remains an unresolved debate around the role of other ectopic fat depots. This includes for example, depots that surround the heart, pancreas, and kidneys, and their contribution to the dysmetabolic state and NAFLD risk, independent of VAT. Perhaps, one of the most hotly argued topics is the role of epicardial adipose tissue (EAT).

EAT is the visceral fat depot of the heart and is considered a metabolically active organ that produces both proinflammatory and anti-inflammatory mediators [5]. EAT amount has high heritability, estimated in twin studies to be up to $80 \%$, with only a modest impact for environmental factors $(20 \%)$ [6]. Notably, ethnic variation is observed for EAT volume; with multiple reports suggesting that Asians tend to have higher EAT compared to Caucasian populations for the same adiposity, as measured by BMI [7, 8]. EAT is relatively

Mohammed Eslam and Jacob George contributed equally to all aspects of this article.

Mohammed Eslam

mohammed.eslam@sydney.edu.au

Jacob George

jacob.george@sydney.edu.au

1 Storr Liver Centre, Westmead Institute for Medical Research, Westmead Hospital and University of Sydney, Westmead, NSW 2145, Australia easily quantified either by echocardiography or computerised tomography. Hence, its assessment could have important clinical implications for the stratification and prediction of cardio-metabolic risk, particularly as EAT is increased in conjunction with a variety of disorders including metabolic syndrome, atherosclerosis and diabetes $[9,10]$.

Interestingly, epicardial and intra-abdominal fat have the same embryonic origin, with both derived from brown adipose tissue [11]. Hence, echocardiographic EAT could be considered a marker of visceral adiposity. On the basis of findings that NAFLD increases the risk of cardiovascular disease independent of confounding metabolic risk factors $[12,13]$, it is conceivable that EAT might play a role in NAFLD development and progression, as well as in increasing NAFLD-associated cardiovascular risk. However, current data on the correlation between EAT and NAFLD is conflicting $[14,15]$.

In many instances, well conducted systematic reviews (with or without meta-analyses) can help to overcome controversies and debates by enabling an objective synthesis of available literature through a meticulous process of careful and systematic identification, critical appraisal and pooling of relevant studies. In the current issue, Liu et al. [16] systematically reviewed the evidence for the association of EAT thickness and volume with NAFLD, and then performed a meta-analysis to evaluate the association. The meta-analysis included thirteen case-control studies ( $n=2260$ patients) and confirmed that EAT was significantly increased in NAFLD patients compared with controls. Among patients with NAFLD, EAT correlated with the severity of hepatic steatosis and fibrosis, and atherosclerotic cardiovascular disease, suggesting a dose-response effect and thereby biological plausibility. Notably, no association was observed between EAT and NASH, but this likely reflects the fact that only two studies in the meta-analysis (comprising just 200 patients), had this data. Thus, this analysis was highly likely underpowered to assess for association. Importantly, the authors' power calculations indicated that at least 1000 


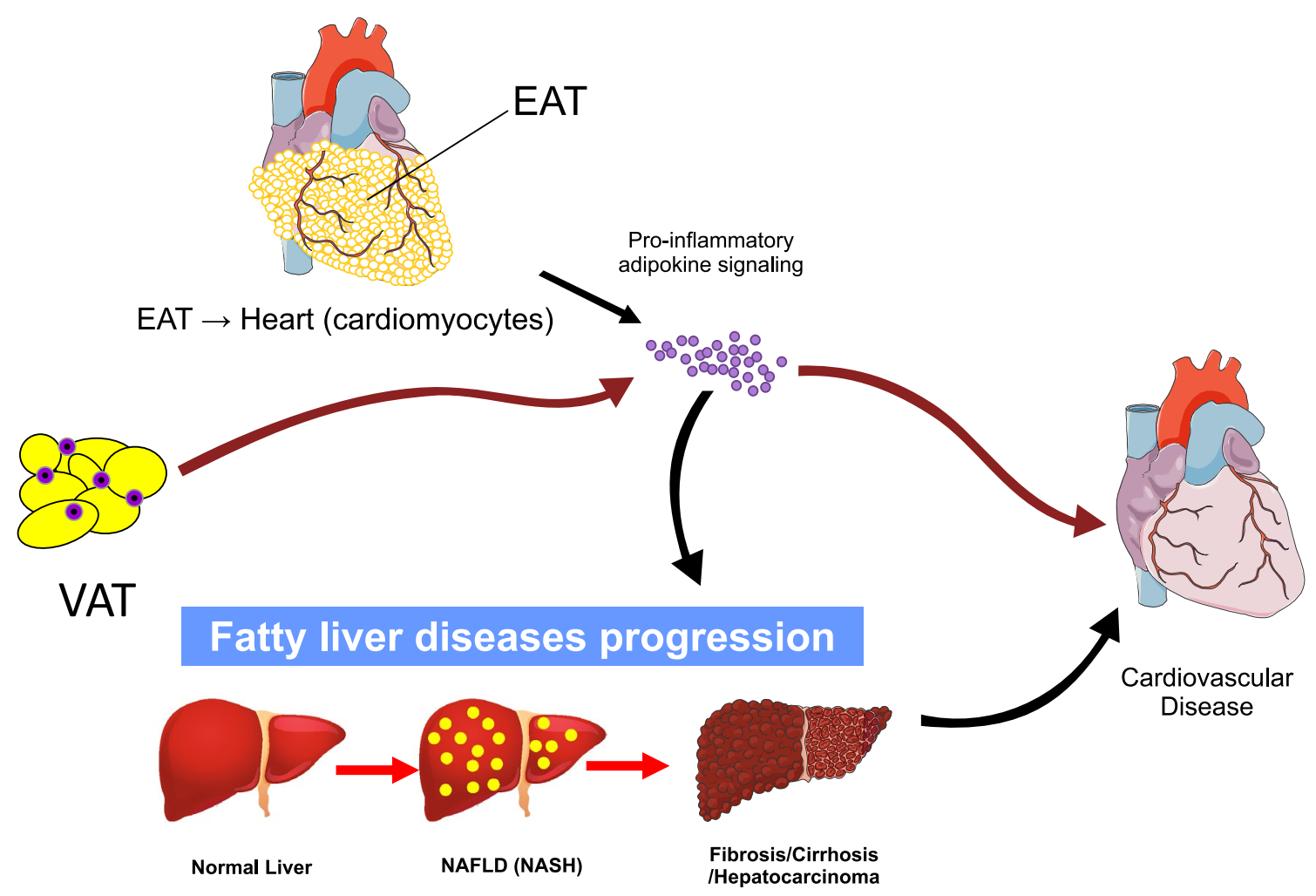

Fig. 1 Role of epicardial and visceral adipose tissue in non-alcoholic fatty liver disease. VAT: Visceral Adipose Tissue, EAT: Epicardial adipose tissue

patients would be required in such an analysis, for a more definitive conclusion. Thus, additional studies are required to examine the link between steatohepatitis with EAT. In addition, the cross-sectional nature of all the included studies influences any assessment of causality and whether EAT precedes NAFLD or vice versa, or in fact if they are both concomitant phenotypes that occur in the context of systemic metabolic dysfunction.

A further critical concern in relation to inferences of causality is that excess EAT is more commonly present associated with visceral obesity, than in isolation without visceral obesity. Thus, when studies do not concomitantly examine both VAT and EAT in relation to NAFLD risk, whether there is any independent contribution of EAT to NAFLD histology, is difficult to ascertain. Further, a previous study has suggested that metabolic syndrome has a greater impact on EAT than NAFLD and that subjects with NAFLD and diabetes tend to have more EAT than those without diabetes. In this regard, it should be noted that adipose secreted proteins (adipokines) and inflammatory mediators, typically act in an autocrine or paracrine manner, with less systemic spill over [17]. In contrast, according to the "portal hypothesis" for VAT, the direct infusion of mediators into the portal circulation and thence to the liver [18], indicates that causal metabolic associations are more likely associated with VAT than EAT. However, more detailed studies need to be undertaken to dissect and clarify this issue (Fig. 1). Notably, recent data suggest that EAT mass and related cardiovascular risk can be attenuated through weight loss, exercise and some pharmaceutical drugs such as lipid-lowering agents, sodium-glucose transporter 2 inhibitors and the glucagon-like peptide 1 analogues [19-23].

In summary, ectopic fat depots and perhaps EAT likely play crucial roles in the development and progression of NAFLD, and increase the risk of cardiovascular diseases in these patients. However, while the data in this report increase current knowledge, more detailed studies need to be undertaken and reported, to characterise the precise roles of EAT as opposed to other ectopic fat depots in the pathogenesis of NAFLD. As with most science, the current report serves as an impetus to undertake more research in order to clarify the questions raised.

Acknowledgements ME and JG are supported by the Robert W. Storr Bequest to the Sydney Medical Foundation, University of Sydney; a National Health and Medical Research Council of Australia (NHMRC) Program Grant (APP1053206, APP1149976) and Project Grants (APP1107178 and APP1108422). 


\section{Compliance with ethical standards}

Conflict of interest Mohammed Eslam and Jacob George declare no competing interests for this manuscript.

Research involving human participants and/or animals Not applicable.

Informed consent Not applicable.

\section{References}

1. Jaacks LM, Vandevijvere S, Pan A, McGowan CJ, Wallace C, Imamura F, et al. The obesity transition: stages of the global epidemic. Lancet Diabetes Endocrinol 2019;7:231-240

2. Younossi Z, Anstee QM, Marietti M, Hardy T, Henry L, Eslam $\mathrm{M}$, et al. Global burden of NAFLD and NASH: trends, predictions, risk factors and prevention. Nat Rev Gastroenterol Hepatol 2018;15:11-20

3. van der Poorten D, Milner KL, Hui J, Hodge A, Trenell MI, Kench JG, et al. Visceral fat: a key mediator of steatohepatitis in metabolic liver disease. Hepatology 2008;48:449-457

4. Perseghin G. Lipids in the wrong place: visceral fat and nonalcoholic steatohepatitis. Diabetes Care 2011;34(Suppl 2):S367-S370

5. Mazurek T, Zhang LF, Zalewski A, Mannion JD, Diehl JT, Arafat $\mathrm{H}$, et al. Human epicardial adipose tissue is a source of inflammatory mediators. Circulation 2003;108:2460-2466

6. Jermendy AL, Kolossvary M, Drobni ZD, Tarnoki AD, Tarnoki DL, Karady J, et al. Assessing genetic and environmental influences on epicardial and abdominal adipose tissue quantities: a classical twin study. Int J Obes 2018;42:163-168

7. El-Khoudary SR, Shin C, Masaki K, Miura K, Budoff M, Edmundowicz D, et al. Ectopic cardiovascular fat in middle-aged men: effects of race/ethnicity, overall and central adiposity: the ERA JUMP study. Int J Obes 2015;39:488-494

8. Adams DB, Narayan O, Munnur RK, Cameron JD, Wong DTL, Talman AH, et al. Ethnic differences in coronary plaque and epicardial fat volume quantified using computed tomography. Int J Cardiovasc Imaging 2017;33:241-249

9. Li YR, Liu B, Li Y, Jing XD, Deng SB, Yan YL, et al. Epicardial fat tissue in patients with diabetes mellitus: a systematic review and meta-analysis. Cardiovasc Diabetol 2019;18:3

10. Jeong JW, Jeong MH, Yun KH, Oh SK, Park EM, Kim YK, et al. Echocardiographic epicardial fat thickness and coronary artery disease. Circ J 2007;71:536-539

11. Marchington JM, Mattacks CA, Pond CM. Adipose-tissue in the mammalian heart and pericardium — structure, fetal development and biochemical-properties. Comp Biochem Physiol B Biochem Mol Biol 1989;94:225-232

12. Adams LA, Lymp JF, St Sauver J, Sanderson SO, Lindor KD, Feldstein A, et al. The natural history of nonalcoholic fatty liver disease: a population-based cohort study. Gastroenterology 2005;129:113-121
13. Vilar-Gomez E, Calzadilla-Bertot L, Wong VWS, Castellanos M, Aller-de-la-Fuente R, Metwally M, et al. Fibrosis severity as a determinant of cause-specific mortality in patients with advanced nonalcoholic fatty liver disease: a multi-national cohort study. Gastroenterology 2018;155:443

14. Psychari SN, Rekleiti N, Papaioannou N, Varhalama E, Drakoulis C, Apostolou TS, et al. Epicardial fat in nonalcoholic fatty liver disease: properties and relationships with metabolic factors, cardiac structure, and cardiac function. Angiology 2016;67:41-48

15. Brouha SS, Nguyen P, Bettencourt R, Sirlin CB, Loomba R. Increased severity of liver fat content and liver fibrosis in non-alcoholic fatty liver disease correlate with epicardial fat volume in type 2 diabetes: a prospective study. Eur Radiol 2018;28:1345-1355

16. Liu B et al. Association of epicardial adipose tissue with nonalcoholic fatty liver disease: a meta-analysis. Hepatol Int 2019. https://doi.org/10.1007/s12072-019-09972-1

17. Villarroya F, Gavalda-Navarro A, Peyrou M, Villarroya J, Giralt $\mathrm{M}$. The lives and times of brown adipokines. Trends Endocrinol Metab 2017;28:855-867

18. Kabir M, Catalano KJ, Ananthnarayan S, Kim SP, Van Citters GW, Dea MK, et al. Molecular evidence supporting the portal theory: a causative link between visceral adiposity and hepatic insulin resistance. Am J Physiol Endocrinol Metab 2005;288:E454-E461

19. Christensen RH, Wedell-Neergaard AS, Lehrskov LL, Legaard GE, Dorph E, Larsen MK, et al. Effect of aerobic and resistance exercise on cardiac adipose tissues: secondary analyses from a randomized clinical trial. JAMA Cardiol 2019;8:778-787

20. Dutour A, Abdesselam I, Ancel P, Kober F, Mrad G, Darmon P, et al. Exenatide decreases liver fat content and epicardial adipose tissue in patients with obesity and type 2 diabetes: a prospective randomized clinical trial using magnetic resonance imaging and spectroscopy. Diabetes Obes Metab 2016;18:882-891

21. Iacobellis G, Mohseni M, Bianco SD, Banga PK. Liraglutide causes large and rapid epicardial fat reduction. Obesity 2017;25:311-316

22. Alexopoulos N, Melek BH, Arepalli CD, Hartlage GR, Chen ZJ, $\mathrm{Kim} \mathrm{S}$, et al. Effect of intensive versus moderate lipid-lowering therapy on epicardial adipose tissue in hyperlipidemic postmenopausal women a substudy of the BELLES trial (beyond endorsed lipid lowering with EBT scanning). J Am Coll Cardiol 2013;61:1956-1961

23. Willens HJ, Byers P, Chirinos JA, Labrador E, Hare JM, de Marchena E. Effects of weight loss after bariatric surgery on epicardial fat measured using echocardiography. Am J Cardiol 2007;99:1242-1245

Publisher's Note Springer Nature remains neutral with regard to jurisdictional claims in published maps and institutional affiliations. 